\title{
Prolonged $\beta$-catenin stabilization and tcf-dependent transcriptional activation in hyperplastic cutaneous wounds
}

\author{
Sophia Cheon ${ }^{1,2}$, Raymond Poon ${ }^{1}$, Chunying Yu ${ }^{1}$, Michael Khoury ${ }^{1}$, Rob Shenker ${ }^{3}$, \\ Joel Fish ${ }^{3}$ and Benjamin A Alman ${ }^{1,2,4}$ \\ ${ }^{1}$ Program in Developmental Biology, The Hospital for Sick Children, Toronto, Ontario, Canada; ${ }^{2}$ Institute of \\ Medical Science, University of Toronto, Toronto, Ontario, Canada; ${ }^{3}$ Ross Tilley Burn Centre, Sunnybrook $\mathcal{E}$ \\ Womens College Health Sciences Centre, Division of Plastic Surgery, University of Toronto, Toronto, Ontario, \\ Canada and ${ }^{4}$ Division of Orthopaedic Surgery, The Hospital for Sick Children and the University of Toronto, \\ Toronto, Ontario, Canada
}

\begin{abstract}
Mesenchymal cells that accumulate during the proliferative phase of wound healing and that are present in hyperplastic wounds share cytologic similarities with the cells from fibroproliferative lesions in which there is activation of $\beta$-catenin-mediated transcription. Re-excision wounds from a previous biopsy and samples from hyperplastic cutaneous wounds were studied along with normal tissues. During normal wound healing, there was an increase in $\beta$-catenin protein level, peaking 4 weeks following the insult and returning towards baseline level by 12 weeks. Hyperplastic wounds exhibited a prolonged duration of elevated $\beta$-catenin, lasting more than 2 years following the initial injury. The level of expression of genes known to be upregulated in the proliferative phase of wound healing ( $\alpha$-smooth muscle actin and type three collagen), correlated with $\beta$-catenin protein level. The phosphorylation level of glycogen synthase kinase-3- $\beta$, a kinase important for $\beta$-catenin protein destabilization, correlated with $\beta$-catenin protein level. $\beta$-Catenin was transcriptionally active in these wounds as demonstrated by the expression of the $\beta$-catenin target genes (MMP-7 and $F M$ ) and by activation of a tcfreporter in primary cell cultures. $\beta$-catenin stabilization increases cell proliferation and motility in fibroblasts in vitro, and likely has a similar function during its transient elevation in the proliferative phase of normal wound healing. In hyperplastic wounds, there is dysregulation of $\beta$-catenin, maintaining the mesenchymal cells in a prolonged proliferative state. As such, $\beta$-catenin likely plays a central role in mesenchymal cells during the healing process, and is an appealing therapeutic target for disorders of wound healing.

Laboratory Investigation (2005) 85, 416-425, advance online publication, 17 January 2005; doi:10.1038/labinvest.3700237
\end{abstract}

Keywords: beta-catenin; fibronectin; transcriptional regulation; wound healing

Healing is a dynamic process involving soluble factors, blood-borne cells, extracellular matrix, and parenchymal cells. Although there is overlap between them, the healing process progresses through three major phases: an initial or inflammatory phase; a proliferative phase; and a remodeling phase. During the proliferative phase, there is reconstitution of the barrier and structural function of the skin. These two functions are associated with the epithelial component and the mesenchymal component (also called stromal or granulation

Correspondence: Dr BA Alman, MD, The Hospital for Sick Children, 555 University Avenue, Toronto, Ontario, Canada M5G 1X8.

E-mail: benjamin.alman@sickkids.ca

Received 14 July 2004; revised 26 November 2004; accepted 2 December 2004; published online 17 January 2005 tissue) of the wound. Re-epithelialization begins within hours after injury, and within 2 days, cells at the wound margin proliferate behind the actively migrating cells. Cells forming the mesenchymal component begin to invade the wound space about 4 days after injury. This tissue is composed of macrophages, fibroblasts, and blood vessels. The fibroblasts produce the new extracellular matrix necessary to support cell ingrowth and to provide strength to the wound. These cells express higher levels of type three collagen and $\alpha$-smooth muscle actin. Once an abundant collagen matrix has been deposited in the wound, the fibroblasts stop producing collagen, and the fibroblast-rich granulation tissue is replaced by a relatively acellular scar. It is during this remodeling phase that extracellularmatrix reorganization takes place. ${ }^{1,2}$ 
The fibroblast-like mesenchymal cells that accumulate beneath the epithelium during the proliferative phase of healing share cytologic similarity with cells from a variety of fibroproliferative processes, such as aggressive fibromatosis, a locally invasive tumor. Aggressive fibromatosis is associated with somatic mutations resulting in the stabilization of $\beta$-catenin protein, and the activation of $\beta$-catenin mediated tcf-dependent signaling. ${ }^{3,4}$ Evidence that $\beta$-catenin stabilization is sufficient to cause aggressive fibromatosis is derived from transgenic mice that express a stabilized form of $\beta$-catenin which develop aggressive fibromatosis tumors. ${ }^{5}$ $\beta$-catenin is a crucial mediator in the canonical WNT (or wingless) signaling cascade. In the absence of an appropriate WNT ligand, glycogen synthase kinase (GSK) $3 \beta$ promotes the phosphorylation of $\beta$-catenin at key Ser/Thr residues, targeting it for degradation through the ubiquitin-ligase pathway. Additional proteins including Adenomatous Polyposis Coli (APC) and Axin act in a multiprotein complex with GSK-3- $\beta$ to regulate $\beta$-catenin levels. In response to an appropriate WNT ligand, the GSK-3- $\beta$ activity is inhibited resulting in an elevation of the protein level of $\beta$-catenin, associated with phosphorylation of GSK-3- $\beta$. $\beta$-Catenin protein can also be stabilized by a mutation in key Ser/Thr phosphorylation sites, or if there is a mutation in one of the proteins in the multi protein complex regulating $\beta$-catenin protein phosphorylation. Stabilized $\beta$-catenin can translocate to the nucleus, and bind to transcription factors in the tcf-lef family, transactivating transcription. ${ }^{6,7}$ Tcf-lef transcription factors regulate gene expression in a cell-type specific manner. As such, the target genes in fibroblasts are not the same as those in epithelial cells. Two genes that are upregulated in mesenchymal cells in response to $\beta$-catenin mediated tcf-dependent transcription are matrix metalloproteinase seven $(M M P-7)$ and fibronectin $(F N) .^{8-10}$ Both matrix metalloproteinases and fibronectin are known to be upregulated during wound healing, and are also expressed in hyperplastic wounds. ${ }^{11-13}$

Fibroblasts present during the proliferative healing phase have a cytological appearance resembling immature fibroblasts with abundant rough endoplasmic reticulum. However, as the healing process proceeds, fibroblasts show a more mature phenotype with prominent microfilament bundles. The gross cytological appearance of the less mature fibroblasts in the proliferative phase of wound healing is quite similar to the appearance of the cells in aggressive fibromatosis. Thus, a role for $\beta$-catenin protein stabilization and tcf-dependent transcriptional activation was investigated in murine wound healing. Using a transgenic tcf-reporter mouse, it was found that $\beta$-catenin protein level is elevated and tcf-dependent transcription is activated in the mesenchymal, fibroblast-like cells during the proliferative phase of wound healing. ${ }^{5}$
Hyperplastic scars are characterized by the persistence of fibrous bundles, which exist in a typical horizontal pattern in the upper dermis and a whorled appearance in the deeper dermis. The dermal layer is composed of cells cytologically reminiscent of the immature fibroblasts present during the proliferative phase of wound healing. Many of the factors upregulated during the proliferative phase of wound healing such as expression of various collagens, $\alpha$-smooth muscle actin, and fibronectin are also upregulated in hyperplastic wounds. The cause of hyperplastic wounds is not known, but factors such as mechanical stress and overactivation of growth factor, such as transforming growth factor $\beta$, are implicated. The similarities in cell cytology and gene expression between hyperplastic wounds and the proliferative phase of normal wound healing suggest that, in some aspects, cells in hyperplasic wounds behave as though they were in a prolonged proliferative phase of healing. ${ }^{2,14,15}$ In this study, we examine the role of $\beta$ catenin during normal human cutaneous wound healing and in hyperplastic cutaneous wounds.

\section{Methods}

\section{Samples}

Patients undergoing resection for a benign musculoskeletal tumor following a previous open biopsy, and patients undergoing surgical excision for a hyperplastic wound were investigated. In the case of patients undergoing surgery after a previous open biopsy, the entire initial wound, including a margin of normal skin, was removed, as is the usual practice for surgical resection of a musculoskeletal tumor following a previous open biopsy. A section of the subcutaneous tissue from a separate sample from the surrounding normal skin, taken as far away as possible from the initial wound, was processed for analysis as a normal skin control. Cases were available for analysis from resections performed 2 weeks (four cases), 4 weeks (five cases), 6 weeks (four cases), 8 weeks (three cases), 12 weeks (four cases), or 6 months (two cases) following the initial open biopsy. In all, 10 cases of hyperplastic wounds that underwent surgery between 1 and 5 years following an initial injury were studied, and tissues processed in a similar manner. Four of these patients had a previous sharp penetrating traumatic event causing a wound in an extremity, one had blunt trauma, and the remainder had a burn injury. On examination, the scars were at least $1.0 \mathrm{~cm}$ wide. None of the patients had previous surgery for a hyperplastic scar. Patients underwent surgery because of the cosmetic appearance of the scar or functional limitations related to the scar. All of the wound samples were obtained from wounds involving the extremities, but not located directly over a joint, to eliminate the possibility that differences found might be related to anatomic site. The size of 
the subcutaneous tissue available from the hyperplastic wounds were smaller than those from the wounds from previous open biopsies, and such, the case material available for analysis was limited. Thus not all of the analyses were able to be undertaken on every sample. In all cases, a section of the wound and normal tissue was processed in an identical manner. Full thickness sections were cryopreserved, and also prepared as formalin-fixed paraffin-embedded materials. Samples were processed and stained with hematoxylin and eosin for histologic analysis.

\section{Protein Analysis}

Proteins were extracted from tissue samples and analyzed using Western analysis as previously reported. ${ }^{3}$ In brief, equal amounts of total proteins were electrophoresed on an SDS-polyacrylamide gel, transferred to a polyvinylidene diflouride membrane, and stained to verify an equal amount of transferred proteins from each sample. Western blot was performed using an antibody to $\beta$-catenin (Transduction Laboratories, Lexington, KY, USA), phosphor-Ser-9 GSK3 $\beta$ (New England Biolabs), actin (Oncogene), and total GSK3 $\beta$ (Transduction Laboratories). Hybridization was carried out overnight at $4^{\circ} \mathrm{C}$ and detected using a peroxidase secondary antibody and chemiluminescence. The same blots were stripped and then reprobed using each antibody. Actin was used as an additional loading control. The Western analysis for each sample was performed in triplicate. The immunoblot results were scanned, and the digital data analyzed on a computer. Densitometry was performed by measuring the density across the band of interest, and normalizing this to the average background density of the blot. The comparison between the control band and band of interest was performed using comparisons made from the same digital data, which contained both bands. The means, standard deviations, and 95\% confidence intervals for the relative density of $\beta$-catenin were determined for samples, and these were compared using the twoway $t$-test.

Immunohistochemistry was performed on formalin-fixed, paraffin-embedded material using a previously reported technique. ${ }^{3,4}$ Tissues were dewaxed and quenched in hydrogen peroxide/methanol solution, immersed in $10 \mathrm{mmol} / \mathrm{l}$ citric acid, and heated for $15 \mathrm{~min}$ in a $750-\mathrm{W}$ microwave oven. After washing and blockade with $1 \%$ serum bovine albumin, the slides were incubated with monoclonal antibody to $\beta$-catenin (Transduction Laboratories) at $10 \mu \mathrm{g} / \mathrm{ml}$ overnight at $40^{\circ} \mathrm{C}$. Tissues were washed with PBS, incubated with a secondary anti-mouse immunoglobulin, and detected using immunoperoxidase staining (Vector Laboratories, Burlingame, CA, USA). Wound and normal tissues were processed at the same time to avoid slight variations in technique that might alter staining characteristics.

\section{RNA Analysis}

Total RNA was extracted from cryopreserved tissues in the re-excision wounds, hyperplastic wounds, and accompanying normal tissues. RNA was extracted using Trizol regents (Life Technologies, Inc.) and converted to cDNA using reverse transcriptase with a poly-T primer. PCR primer pairs that amplify a product that crosses introns were chosen from previous publications ${ }^{16-20}$ for reduced glyceraldehyde-phosphate dehydrogenase (GAPDH), $\beta$-catenin, genes known to be upregualted in the proliferative phase of wound healing ( $\alpha$-smooth muscle actin and type three collagen), and the $\beta$ catenin target genes $M M P-7$ and FN. PCR was performed under semiquantitative conditions as previously reported..$^{18}$ Each sample was amplified for several different cycle numbers, after confirming amplification in the linear range, using the primer pairs for the gene of interest, as well as for the housekeeping control gene, GAPDH. Resultant products were electrophoresed, stained with ethidium bromide, and photographed under ultraviolet light. Densitometry was utilized to determine the relative difference in density of the resultant band of interest compared to the band from amplification using primers to the control gene, GAPDH. The PCR results were scanned, and the digital data analyzed on a computer. Densitometry was performed by measuring the density across the band of interest, and normalizing this to the average background density. The comparison between the control band and band of interest was performed using comparisons made from the same digital data, which contained both bands from a single blot. The data were considered as a ratio between the gene of interest and the control gene. The PCR reactions for each sample were performed in triplicate for primer pair. There was less than $10 \%$ variation in the density ratios between the gene of interest and the control gene for the triplicates for each specimen. The means, standard deviations, and 95\% confidence intervals for the relative density for each gene of interest was determined for each sample relative to the level of $G A P D H$, and these were compared between samples using the two-way $t$-test.

\section{Primary Cell Cultures and tcf-Reporter Assay}

Primary cell cultures from five re-excision wounds, from three hyperpastic wounds, and from the associated surrounding normal dermal tissues were established using an explant technique..122 Cells were examined for tcf-dependent transcription after their first passage in culture. The cells plated on 35$\mathrm{mm}$ dishes were transiently transfected in triplicate with $1 \mu \mathrm{g}$ of the tcf-lef luciferase reporter construct 
pTOPFLASH or the control reporter, pFOPFLASH, which contains a mutant tcf-lef consensus binding sequence. Superfect transfection reagent (Qiagen) was utilized according to the manufacturer's instructions, and in all cases, a Rous sarcoma virus $\beta$-galactosidase expression vector was used as a control for transfection efficiency. Cells were harvested $24 \mathrm{~h}$ after transfection, and luciferase enzyme activity was measured using a luminometer, and normalized to $\beta$-galactosidase activity. A ratio of the normalized pTOPFLASH/pFOPFLASH luminescence was calculated for each cell dish. ${ }^{21,23,24}$ The experiment was performed in triplicate. The mean, standard deviation, and 95\% confidence interval of the result from the culture derived from the wound tissues were compared to the results from the cell culture derived from normal tissue, using the two-way $t$-test.

\section{Results}

\section{$\beta$-Catenin Protein is Transiently Elevated during the Proliferative Phase of Wound Healing}

We analyzed $\beta$-catenin protein levels in healing wounds from patients undergoing re-excision for a tumor. There was a robust dermal mesenchymal component on histological evaluation to the samples from the re-excision wounds, consistent with the appearance of the proliferative phase of cutaneous wound healing. The fibroblasts present during the proliferative healing phase resemble immature fibroblasts with a highly synthetic appearance. In the later phase of healing, the fibroblasts show a more mature phenotype with prominent microfilament bundles (Figure 1). We examined the level of expression of $\alpha$-smooth muscle actin and type three collagen, genes known to be upregulated during the proliferative phase of wound healing, in these samples using semi-quantitative RT-PCR. Both genes were expressed at higher levels in the wounds than in surrounding normal tissues, with expression level peaking in wounds 4 weeks following the initial biopsy, and gradually declining towards baseline at 12 weeks following the biopsy (Figure 2). This expression pattern is consistent with the proliferative phase of wound healing occurring 4 weeks following the initial insult. Using Western analysis, the protein level of $\beta$-catenin was compared between wound samples from different times after the initial biopsy. $\beta$-Catenin protein level was elevated in all of the wounds compared to surrounding normal tissues, but the degree of elevation varied with the time course. At 1 week, there was a mild elevation in protein level, the protein level of $\beta$ catenin peaked 4 weeks following the initial injury, and by 12 weeks following the initial insult, levels returned to near baseline (Figure 3).

Immunohistochemistry showed that $\beta$-catenin was located in the cytoplasm and nucleus in the mesenchymal dermal cells in the re-excision samples (Figure 4). Although there was staining for $\beta$-catenin in the epithelial cells, the staining was localized to the cell membranes. This staining pattern suggests that $\beta$-catenin is transcriptionally active in the mesenchymal cells, but not in the epithelial cells. The intensity of $\beta$-catenin staining correlated with the Western analysis results, with normal wounds showing maximal staining 4 weeks following the initial injury. Although it is difficult to show in an objective manner, the less mature fibroblasts seemed to have more beta-catenin staining.

\section{$\beta$-Catenin Protein Elevation is not Associated with a Change in mRNA Level, but Does Correlate with Phospho-Ser-9 GSK-3 $\beta$}

To determine the mechanism responsible for causing an elevation in $\beta$-catenin protein in the wounds, we examined its mRNA level, and the activation of the multiprotein complex responsible for its a

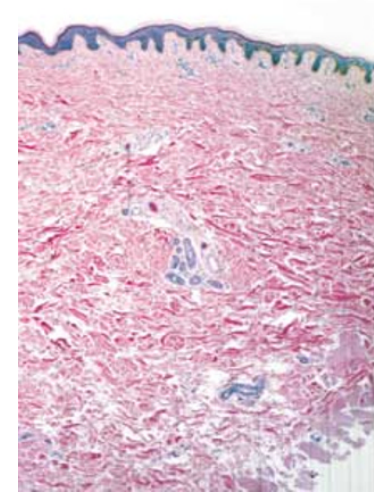

b

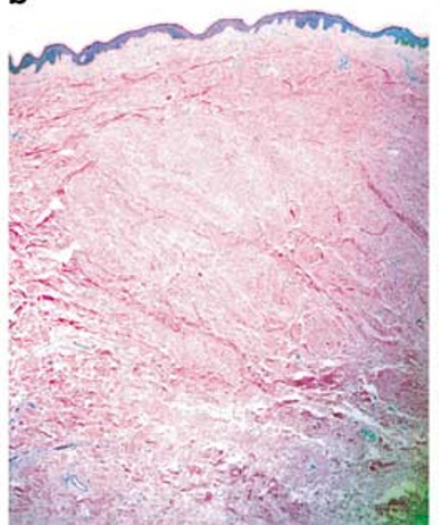

c

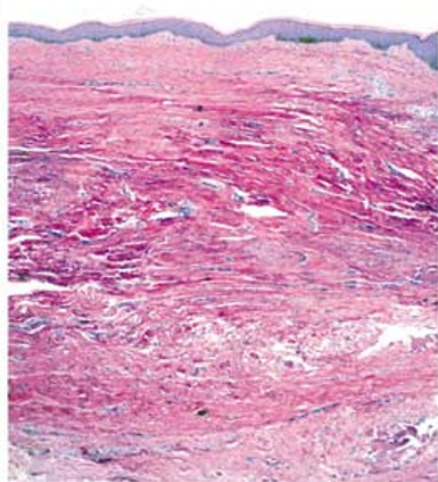

d

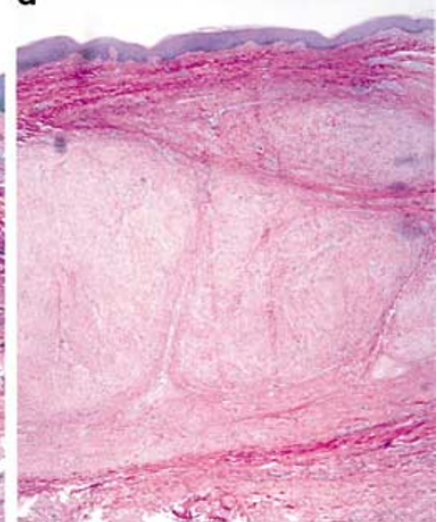

Figure 1 Histologic appearance of cutaneous wounds. Hematoxylin and eosin stained sections $\times 100$. Compared to normal skin (panel a) re-excision wounds showed abundant fibroblast like cells in the mesenchymal component of the wound 4 weeks following injury (panel b). At 12 weeks following the initial biopsy, the size of the mesenchymal component is smaller (panel c), consistent with a switch to the remodeling phase of healing. Hyperplastic wounds maintain a large mesenchymal component to the wound for several years following the initial injury (panel d). 




b Relative expression compared
to the control gene GAPDH
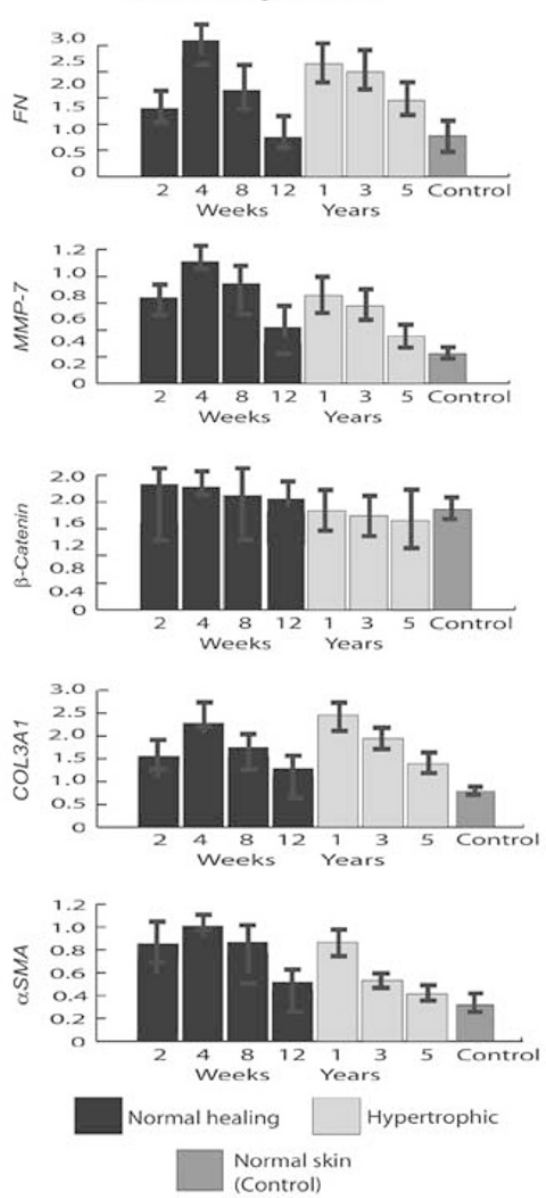

Figure 2 Semiquantitative RT-PCR results for the expression of $F N, M M P-7, \alpha$-smooth muscle actin ( $\alpha S M A$ ), type three collagen (COL3A1), and $\beta$-catenin. Panel a shows a representative PCR result from a normally healing wound 4 weeks following the initial insult. There is a significant increase in FN, MMP-7, $\alpha$ smooth muscle actin, and type three collagen expression 4 weeks following the insult, but no change in $\beta$-catenin mRNA level compared to that of the control, normal tissue. GAPDH expression is used as a control. Panel b shows relative expression of the various genes at different time points following the initial insult in normal wound healing as in hyperplastic wounds, compared to the control gene, GAPDH. The value for normal skin is given in the last bar in the graph. Error bars are 95\% confidence intervals.

ubiquitin-mediated degradation. The mRNA level of $\beta$-catenin was investigated using semiquantitative RT-PCR, and no substantial difference in expression level over the various time points examined was detected (Figure 2). GSK-3- $\beta$ is a member of the multiprotein complex that targets $\beta$-catenin for ubiquitin-mediated degradation. Although the mechanism by which GSK-3- $\beta$ regulates $\beta$-catenin is not completely elucidated, WNT signaling activation causes inactivation of GSK-3- $\beta$, which is associated with its phosphorylation at Ser-9. We examined the protein level of Phopho-Ser-9-GSK-3- $\beta$ compared to total GSK-3- $\beta$, and found that level of Phopho-Ser-9-GSK-3- $\beta$ correlated with the protein a

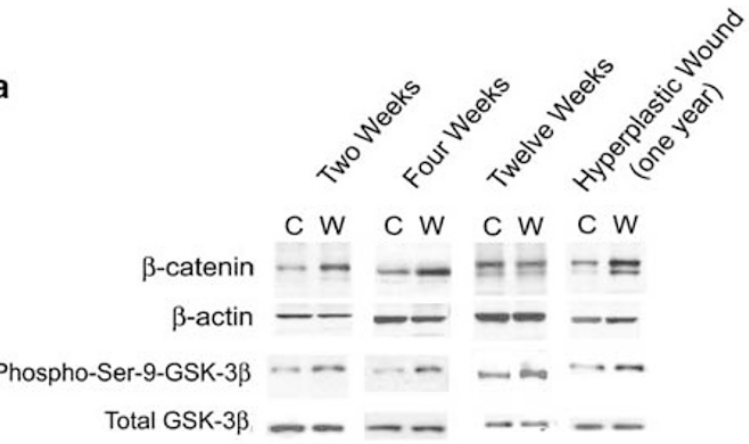

b


Figure 3 Western analysis showing a transiently elevated level of $\beta$-catenin in the normally healing and a prolonged elevation in hyperplastic wounds, and a transient increase in the level of phospho-Ser-9-GSK-3- $\beta$. Equal amounts of protein from a wound and normal tissues are loaded in each comparison. The blots were also probed using an antibody to $\beta$-catenin, Phospho-Ser-9-GSK$3-\beta$, total GSK-3- $\beta$, and $\beta$-actin as an additional loading control. Panel a shows Western analysis results from representative samples. Lanes labeled 'W' are loaded with protein extracted from the wounds and lanes labeled ' $\mathrm{C}$ ' are loaded with extract from the normal tissues from the same patient. There is an elevation in $\beta$-catenin protein level in the normally healing wounds that peaks 4 weeks following the initial insult, and which declines to baseline levels at 12 weeks. In the 1-year-old hyperplastic wound, there is an elevation in $\beta$-catenin protein level comparable to that seen in the normally healing wounds 4 weeks following the initial injury. Phospho-Ser-9-GSK-3- $\beta$ levels mirror the $\beta$-catenin protein level. Panel b shows the relative densities of the band corresponding to $\beta$-catenin as a ratio to the band corresponding to $\beta$-actin at various time points in normal and hyperplastic wounds, and the relative ratio of phospho-Ser-9GSK-3- $\beta$ level to total GSK-3- $\beta$ level compared to the ration for the normal tissues. For the normal wounds time points are in weeks, while for the hyperplastic wounds time points are in years. The error bars are $95 \%$ confidence intervals.

level of $\beta$-catenin (Figure 3 ). This suggests that $\beta$-catenin protein is stabilized in the wound samples through a post-transcriptional mechanism, involving GSK-3- $\beta$. 

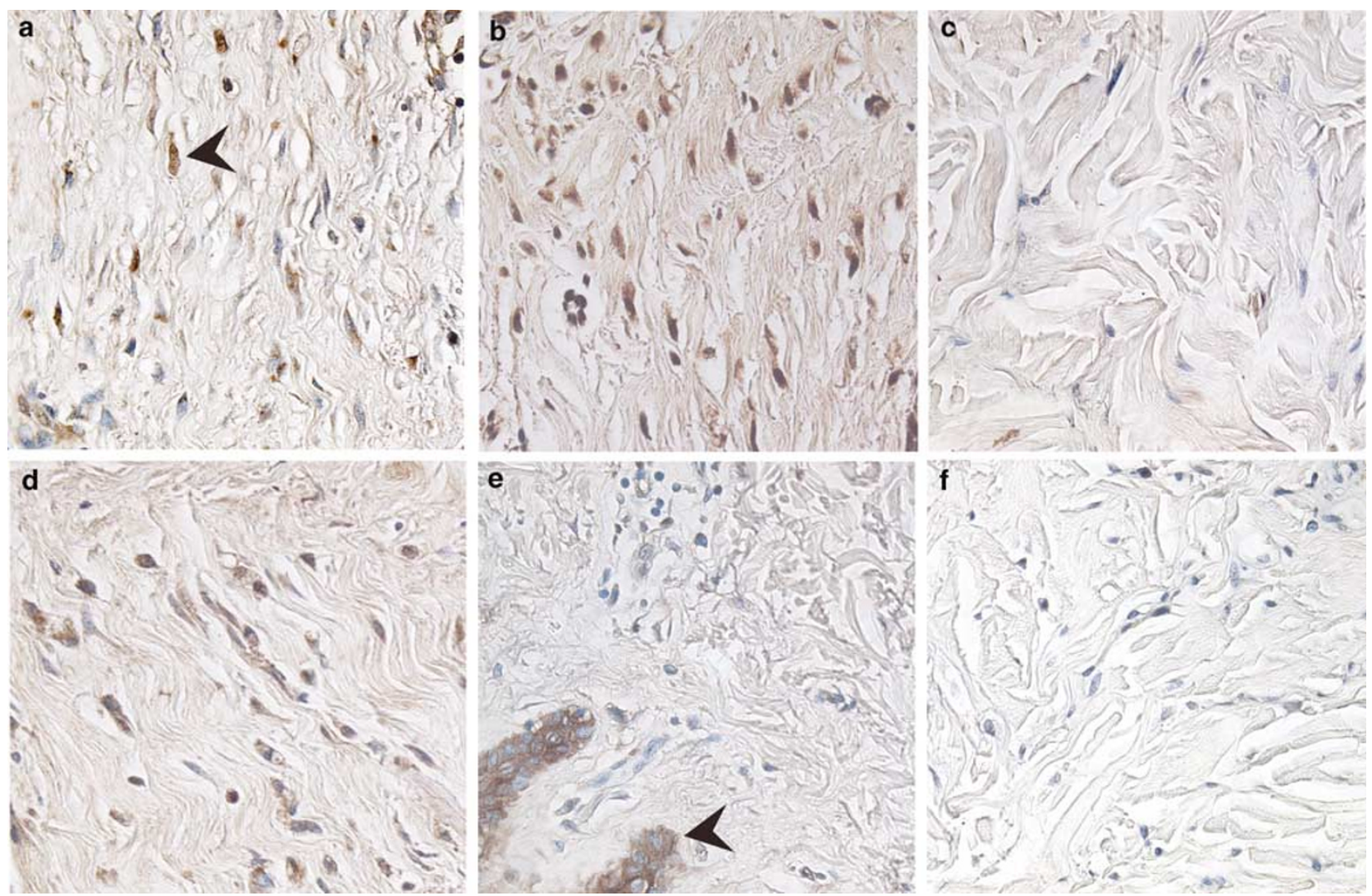

Figure 4 Immunohistochemistry for $\beta$-catenin in wound healing. A robust staining of mesenchymal cells is shown in the normally healing at 2 and 4 weeks, and in the hyperplastic wound compared to unwounded skin. At 2 weeks following injury (panel a) there is staining for $\beta$-catenin, located both in the cell cytoplasm and nucleus in the mesenchymal cells from the healing wound. A representative positively stained cell is labeled with an arrow. The staining is more intense at 4 weeks following injury (pannel b), and declines substantially at 12 weeks following the injury (panel c). The tissue from a hyperplastic wound continues to demonstrate elevated staining even more than 2 years following the initial injury (panel d). A section of normal skin is shown in panel e. The arrow points to positive staining of the epithelial cells. A control section, without the primary antibody, is shown from a 4-week-old wound in panel f.

\section{Tcf-Dependent Transcription is Activated during Wound Healing}

Tcf-dependent transcription was active in the wound tissue as demonstrated by an elevation in tcf-dependent transcriptional activity that was detected using reporter constructs transfected into primary cell cultures. Tcf activation (TOPFLASH) was compared to a control reporter construct (FOPFLASH), in which the tcf-lef consensus binding motif is mutated. In normal tissues, the level of tcf-dependent transcriptional activation was comparable to that found in previous reports (pTOPFLASH/pFOPFLASH ratios averaged 1.2). ${ }^{21,25}$ For the wound fibroblasts, the pTOPFLASH/pFOPFLASH ratios averaged 2.6 (Figure 5), a difference that was statistically significant $(P<0.005)$.

To determine if $\beta$-catenin-mediated tcf-dependent transcription was activated in vivo, we examined expression of two $\beta$-catenin target genes, $F N$ and $M M P$-7. Since $\beta$-catenin target genes vary with the specific tissue type, we selected these genes as they are known to be upregulated in fibroblast-like cells by $\beta$-catenin stabilization. ${ }^{8,10}$ We found that $F N$ and MMP-7 were elevated in the cells during the proliferative phase of wound healing. The relative level of expression was elevated four-fold for $F N$ and three-fold for MMP-7 over baseline returned to that in control tissues by 12 weeks following the initial injury (Figure 2).

\section{Hyperplastic Wounds Exhibit a Prolonged Phase of Elevated $\beta$-Catenin Protein and tcf-Dependent Transcriptional Activation}

Hyperplastic wounds showed a typical histological appearance with spherical aggregates of collagen fibers and fibroblasts oriented in whorls (Figure 1). We examined the level of expression of $\alpha$-smooth muscle actin and type three collagen genes in these wounds, and found the level of expression of $\alpha$ smooth muscle actin to be twice that of the normal tissues, and type three collagen expression to be twice that of normal tissues. This is consistent with previous studies showing upregulation of these genes in hyperplastic wounds. The protein level of $\beta$-catenin in the hyperplastic wounds was comparable to that seen in normal wounds 4 weeks following an initial injury, despite being several 


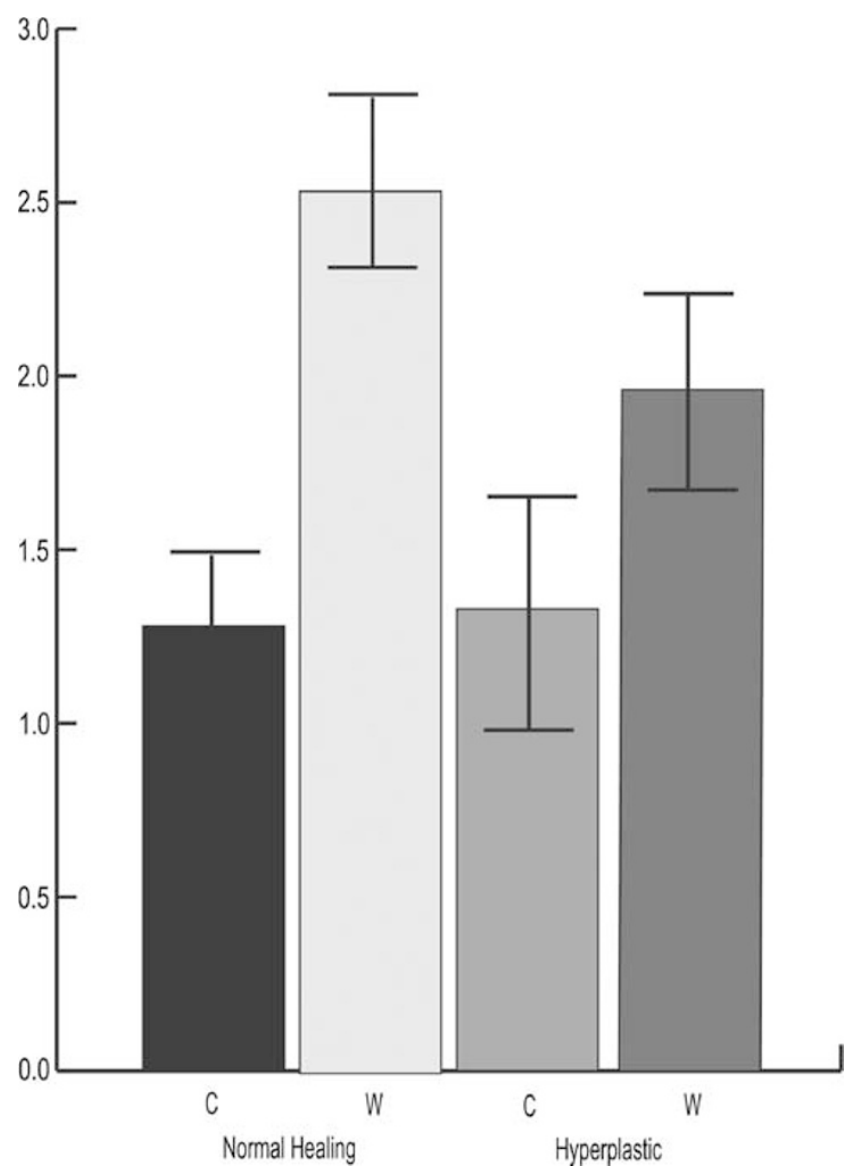

Figure 5 Tcf reporter activation in wound healing. pTOPFLASH/ pFOPFLASH ratios for primary cell cultures derived from normal tissues and from the wounds. A significant increase in this ratio is noted for primary cell cultures obtained from wounds 4 weeks following the initial insult, as well as in the hyperplastic wounds compared to normal tissues. Error bars are $95 \%$ confidence intervals.

years following the initial injury. Although the level of $\beta$-catenin remained elevated for several years following the initial injury, there was a gradual decline in the level over time (Figure 3). Similar to our findings in normal wound healing, the level of Phopho-Ser-9-GSK-3- $\beta$ correlated with the protein level of $\beta$-catenin. Immunohistochemistry revealed a staining pattern for $\beta$-catenin that was similar to than seen in the wounds 4 weeks following the initial surgery, with intense staining located throughout the cell (Figure 4).

To determine if $\beta$-catenin is transcriptionally active in the hyperplastic wounds, the expression of the target genes, $F N$ and $M M P-7$, were examined in the samples, and primary cell cultures were examined for the level of activation of the tcfdependent reporter. We found an elevated level of expression of $F N$ and $M M P-7$ in all the hyperplastic wounds, and found that the relative level of $F N$ and MMP-7 expression correlated with the level of $\beta$-catenin protein, showing a gradual decline with time (Figure 2). Using the tcf-reporter, we found that the pTOPFLASH/pFOPFLASH ratio averaged 2.0 in the hyperplastic wound primary cultures when compared to normal tissues exhibiting a ratio of $1.2, P<0.05$ (Figure 5). This is a slightly lower level of transcriptional activation than in cells during the proliferative phase of normal wound healing, but the relatively small numbers of samples analyzed makes definitive statistical analysis difficult.

\section{Discussion}

We found a transient elevation of $\beta$-catenin protein in dermal mesenchymal cells during the proliferative phase of wound healing, with a peak 4 weeks following the initial injury and a return to baseline 12 weeks following the injury. Furthermore, $\beta$ catenin is transcriptionally active during wound healing, as demonstrated by activation of a reporter construct in primary cell cultures, and by upregulation of the $\beta$-catenin target genes, FN and MMP-7. During the proliferative phase of wound healing, there is transient activation of $\beta$-catenin-mediated tcf-dependent transcription in mesenchymal dermal cells, a cellular signaling process that regulates fibroblast cell proliferation and motility, cell behaviors that are essential for successful healing.

Dermal fibroblasts from hyperplastic wounds did not show a level of $\beta$-catenin protein that was higher than that achieved during the normal wound healing process. However, there was an extended duration of protein elevation. This prolonged elevation of $\beta$-catenin protein was also associated with a prolonged expression of target genes, suggesting that $\beta$-catenin continues to be transcriptionally active throughout this period. Furthermore, there was evidence of continued tcf-dependent transcriptional activation in primary cell cultures from the hyperplastic wounds. The prolonged phase of $\beta$-catenin protein elevation may indicate that the mesenchymal cells are behaving as though there is a prolonged active proliferative phase of wound healing. This could be responsible for the larger size of the mesenchymal component to the hyperplastic wounds, and the relative lack of organized remodeling. $\beta$-Catenin levels eventually return towards baseline in the hyperplastic wounds, but not until several years following the initial injury (Figure 6). After several years, the hyperplastic wounds become relatively acellular, and the relative decline in the number of active mesenchymal cells present likely contributes to the decline in $\beta$-catenin protein level. Although there is similarity in behavior between the acute healing wounds and the hyperplastic wounds in terms of the level of tcf-dependent transcriptional activation, $\beta$-catenin protein stabilization, and expression of target genes, the hyperplastic wounds showed a trend towards lower levels of tcf-dependent transcriptional activation, lower levels of $\beta$-catenin protein, and lower levels of expression of target genes. This finding is consistent 


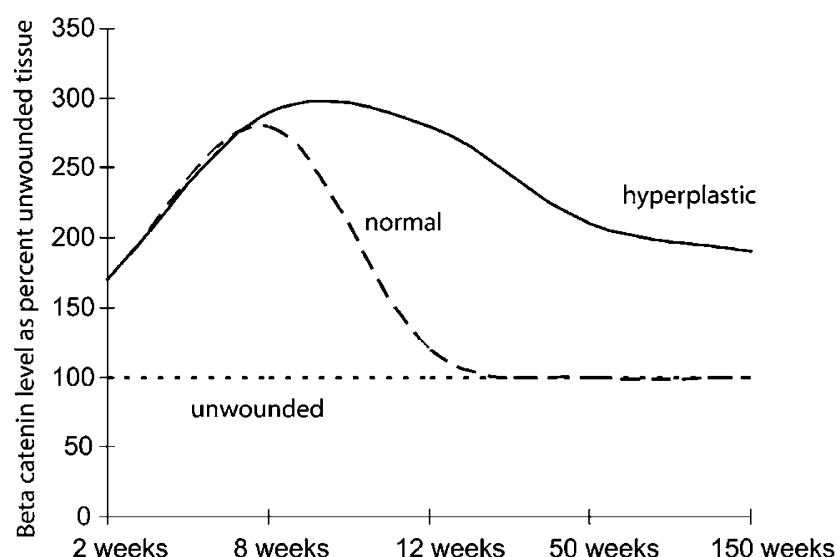

Figure 6 Relative protein level of $\beta$-catenin over time in wound healing and hyperplastic wounds. The normal rise and fall of $\beta$-catenin protein in normal wound healing is dysregulated in hyperplastic wounds, which have a significantly prolonged duration of elevated $\beta$-catenin protein level.

with the notion that $\beta$-catenin-mediated transcription slowly declines with time in the hyperplastic wounds.

During the proliferative phase of wound healing, mesenchymal, dermal cells accumulate beneath the regenerated epithelial layer. These cells need to proliferate and move through the extracellular environment to produce the healing dermal layer. Studies in cell cultures show that $\beta$-catenin protein elevation causes an increased ability of fibroblasts to proliferate, move, and invade through an extracellular matrix. ${ }^{17,22,26} \beta$-Catenin likely functions in dermal fibroblasts to allow them to increase their proliferation and motility rate, in this way acting like activated healing cells. In the case of hyperplastic wounds, the prolonged period of $\beta$-catenin protein elevation, likely maintains the higher rate of proliferation and motility in the dermal cells, thus resulting in a larger size to the dermal component of the wound, but impedes the start of the remodeling phase, which is associated with reduced $\beta$-catenin levels.

$\alpha$-Smooth muscle actin and type three collagen are known to be upregulated during the proliferative phase of wound healing, and in hyperplastic wounds. ${ }^{2,14,15}$ Our expression studies confirmed this finding, in our wound samples, and showed that $\beta$ catenin protein level is highest at times when $\alpha$ smooth muscle actin and type three collagen are also maximally expressed. Since $\beta$-catenin is expressed at high levels at the same time that these genes, which are known to be expressed during the fibrotic response in wound healing, are upregulated, it suggests that $\beta$-catenin plays an important role in the fibroblastic response during wound healing. It also raises the possibility that $\beta$-catenin-mediated transcription plays a role in the regulation of expression of $\alpha$-smooth muscle actin and type three collagen, or that $\alpha$-smooth muscle actin or the extracelleular environment plays a role in regulating the stabilization of $\beta$-catenin. Further studies need to be performed to determine if there is an interaction between $\beta$-catenin, $\alpha$-smooth muscle actin, and type three collagen.

Fibronectin is expressed during the proliferative phase of wound healing, where it plays a crucial role in the formation of the healing granulation tissue, and the levels of fibronectin mRNA are elevated in hyperplastic wounds. ${ }^{27-29}$ Fibronectin exists as several splice variants, several of which are expressed during early development and again in wound healing. We did not investigate the expression of the various variants, but instead used primer pairs that amplified all of the fibronectin variants (sometimes called FN-C). Fibronectin splice variants that are expressed during early development are upregulated during wound healing, suggesting a switch to a less mature cellular phenotype. Fibronectin is thought to act in the extracellular matrix as a conduit for cell migration, especially for epithelial cells during wound healing. ${ }^{28}$ Its elevated expression in hyperplastic wounds may promote the continued activation of cell motility required to maintain the hyperplastic phenotype, and its downregulation may be important in the initiation of the remodeling phase of healing. As such, its elevated expression may help maintain the immature structure to hyperplastic wounds. Although other soluble factors have been implicated in the regulation of fibronectin expression, our data, in concert with previous data on the regulation of fibronectin by $\beta$ catenin, ${ }^{8,9}$ suggests that $\beta$-catenin-mediated tcfdependent expression plays an role in the regulation of fibronectin expression during wound healing. Perhaps it acts to maintain, or perhaps enhance, the level of expression of fibronectin throughout the proliferative phase of healing.

The lack of differences in $\beta$-catenin mRNA despite differences in its protein level, suggest that $\beta$ catenin is regulated by a post-transcriptional mechanism during wound healing. The correlation of Phopho-Ser-9-GSK-3- $\beta$ levels with $\beta$-catenin levels in both normal and hyperplastic wound healing suggests that the multiprotein complex targeting $\beta$-catenin for ubiquitin-mediated degradation is responsible for the stabilization of $\beta$-catenin protein. This could be due to expression of Wnt ligands, or by direct regulation of this multiprotein complex by other factors liberated during wound healing. There are a variety of growth factors and cytokines that are liberated by damaged cells in the early phase of injury. Some of these factors, such as transforming growth factor $\beta$, are implicated in the regulation of $\beta$-catenin protein in vitro. ${ }^{17}$

The switch to turn off $\beta$-catenin at the end of the proliferative phase has yet to be elucidated. However, this 'off switch' seems to be dysregulated in hyperplastic wounds, maintaining the mesenchymal cells in a proliferative healing state for a longer duration of time. Our data on GSK-3- $\beta$ phosphorylation suggests that there is a similar mechanism 
regulating $\beta$-catenin in the hyperplastic wounds as in the proliferative phase of normal healing. Factors that activate $\beta$-catenin in the normal wound healing may continue to be active in these hyperplastic wounds. Mechanical factors may also play a role, perhaps by maintaining the fibroblasts in a more proliferative state, which could be associated with higher levels of $\beta$-catenin. The actual mechanism responsible for the prolonged activation of $\beta$-catenin in hyperplastic wounds remains to be elucidated.

An important role for $\beta$-catenin in fibrous proliferations was initially suggested by the demonstration that $\beta$-catenin becomes elevated in the tumor, aggressive fibromatosis, due to somatic mutations in either $\beta$-catenin itself, or in members of the multiprotein complex regulating its degradation., ${ }^{3,4}$ Subsequently, $\beta$-catenin protein elevation has been demonstrated in other fibroproliferative disorders, such as Dupuytren contracture, but without the demonstration of somatic mutations. ${ }^{30,31}$ The demonstration of transient $\beta$-catenin protein elevation during the proliferative phase of wound healing, and its prolonged duration of elevation in hyperplastic wounds, suggests a common role for $\beta$ catenin in the regulation of cells that compose various fibrous proliferations.

Hyperplastic wounds and disorders of insufficient wound healing are the cause of considerable morbidity. $^{2}$ Our demonstration of a prolonged elevation of $\beta$-catenin protein level in hyperplastic wounds suggests that blockade of $\beta$-catenin, or the modulation of some of the $\beta$-catenin target genes could be developed into a strategy to treat hyperplastic wounds. Insufficient wound healing is associated with a lack of an appropriate mesenchymal component to wound healing. Strategies to increase $\beta$-catenin in these cells may have the potential to improve wound healing in such cases.

\section{Acknowledgements}

This work was funded by the Canadian Institute for Health Research. BAA is supported by the Canadian Research Chairs Program.

\section{References}

1 Diegelmann RF, Evans MC. Wound healing: an overview of acute, fibrotic and delayed healing. Front Biosci 2004;9:283-289.

2 Singer AJ, Clark RA. Cutaneous wound healing. N Engl J Med 1999;341:738-746.

3 Alman BA, Li C, Pajerski ME, et al. Increased betacatenin protein and somatic APC mutations in sporadic aggressive fibromatoses (desmoid tumors). Am J Pathol 1997;151:329-334.

4 Tejpar S, Nollet F, Li C, et al. Predominance of betacatenin mutations and beta-catenin dysregulation in sporadic aggressive fibromatosis (desmoid tumor). Oncogene 1999;18:6615-6620.
5 Cheon SS, Cheah AY, Turley S, et al. Beta-catenin stabilization dysregulates mesenchymal cell proliferation, motility, and invasiveness and causes aggressive fibromatosis and hyperplastic cutaneous wounds. Proc Natl Acad Sci USA 2002;99:6973-6978.

6 Giles RH, van Es JH, Clevers H. Caught up in a Wnt storm: Wnt signaling in cancer. Biochim Biophys Acta 2003;1653:1-24.

7 Lustig B, Behrens J. The Wnt signaling pathway and its role in tumor development. J Cancer Res Clin Oncol 2003;129:199-221.

8 Denys $\mathrm{H}$, De Wever O, Nusgens B, et al. Invasion and MMP expression profile in desmoid tumours. Br J Cancer 2004;90:1443-1449.

9 Gradl D, Kuhl M, Wedlich D. The Wnt/Wg signal transducer beta-catenin controls fibronectin expression. Mol Cell Biol 1999;19:5576-5587.

10 Howard JC, Varallo VM, Ross DC, et al. Elevated levels of beta-catenin and fibronectin in three-dimensional collagen cultures of Dupuytren's disease cells are regulated by tension in vitro. BMC Musculoskelet Disord 2003;16:4-16.

11 Kischer CW, Hendrix MJ. Fibronectin (FN) in hypertrophic scars and keloids. Cell Tissue Res 1983;231: 29-37.

12 Tsou R, Cole JK, Nathens AB, et al. Analysis of hypertrophic and normal scar gene expression with cDNA microarrays. J Burn Care Rehabil 2000;21: 541-550.

13 Soo C, Shaw WW, Zhang X, et al. Differential expression of matrix metalloproteinases and their tissue-derived inhibitors in cutaneous wound repair. Plast Reconstr Surg 2000;105:638-647.

14 Lee JY, Yang CC, Chao SC, et al. Histopathological differential diagnosis of keloid and hypertrophic scar. Am J Dermatopathol 2004;26:379-384.

15 Tuan TL, Nichter LS. The molecular basis of keloid and hypertrophic scar formation. Mol Med Today 1998;4:19-24.

16 McKenna GJ, Chen Y, Smith RM, et al. A role for matrix metalloproteinases and tumor host interaction in hepatocellular carcinomas. Am J Surg 2002;183: 588-594.

17 Cheon SS, Nadesan P, Poon R, et al. Growth factors regulate beta-catenin-mediated TCF-dependent transcriptional activation in fibroblasts during the proliferative phase of wound healing. Exp Cell Res 2004; 293:267-274.

18 Hopyan S, Gokgoz N, Bell RS, et al. Expression of osteocalcin and its transcriptional regulators corebinding factor alpha 1 and MSX2 in osteoid-forming tumours. J Orthop Res 1999;17:633-638.

19 Denys H, De Wever O, Nusgens B, et al. Invasion and MMP expression profile in desmoid tumours. Br J Cancer 2004;5:1443-1449.

20 Hildebrand KA, Zhang M, van Snellenberg W, et al. Myofibroblast numbers are elevated in human elbow capsules after trauma. Clin Orthop 2004;419: 189-197.

21 Tejpar S, Li C, Yu C, et al. Tcf-3 expression and betacatenin mediated transcriptional activation in aggressive fibromatosis (desmoid tumour). Br J Cancer 2001; 85:98-101.

22 Li C, Bapat B, Alman BA. Adenomatous polyposis coli gene mutation alters proliferation through its betacatenin-regulatory function in aggressive fibromatosis (desmoid tumor). Am J Pathol 1998;153:709-714. 
23 Morin PJ, Sparks AB, Korinek V, et al. Activation of beta-catenin-Tcf signaling in colon cancer by mutations in beta-catenin or APC. Science 1997;275: 1787-1790.

24 Korinek V, Barker N, Morin PJ, et al. Constitutive transcriptional activation by a beta-catenin-Tcf complex in APC-/- colon carcinoma. Science 1997;275: 1784-1787.

25 Meng X, Poon R, Zhang X, et al. Suppressor of fused negatively regulates beta-catenin signaling. J Biol Chem 2001;276:40113-40119.

26 Soler C, Grangeasse C, Baggetto LG, et al. Dermal fibroblast proliferation is improved by beta-catenin overexpression and inhibited by E-cadherin expression. FEBS Lett 1999;442:178-182.

27 Muro AF, Chauhan AK, Gajovic S, et al. Regulated splicing of the fibronectin EDA exon is essential for proper skin wound healing and normal lifespan. J Cell Biol 2003;162:149-160.

28 McClain SA, Simon M, Jones E, et al. Mesenchymal cell activation is the rate-limiting step of granulation tissue induction. Am J Pathol 1996;149:1257-1270.

$29 \mathrm{Fu} \mathrm{X}$, Yang Y, Sun T, et al. Comparative study of fibronectin gene expression in tissues from hyperplastic scars and diabetic foot ulcers. Chin Med Sci J 2002;17:90-94.

30 Varallo VM, Gan BS, Seney S, et al. Beta-catenin expression in Dupuytren's disease: potential role for cell-matrix interactions in modulating beta-catenin levels in vivo and in vitro. Oncogene 2003;22: 3680-3684.

31 Montgomery E, Lee JH, Abraham SC, et al. Superficial fibromatoses are genetically distinct from deep fibromatoses. Mod Pathol 2001;14:695-701. 